left ventricle to normal size, during which the reduction of the left ventricular diameter exhibits benefits in recovering left ventricular function in accordance with the concept proposed by Batista. ${ }^{5}$

The possibility of compromise ventricular stroke volume is always present. However, if we consider the base of the papillary muscle as a mark to limit our ventricular reduction, this risk becomes very low, as demonstrated in the echocardiographic results of our latest patient obtained 7 months postoperatively, which present a normal ventricular cavity and normal ventricular ejection fraction.

Although this study involved few patients, this simple technical variation could be used to close the ventricular septal rupture without any residual shunt and could represent one more alternative to resolve a difficult surgical problem.

\section{References}

1. Skillington PD, Lamb RD, Monro JL, et al. Surgical treatment of infarct related ventricular septal defects: improved early results combined with analysis of late functional status. J Thorac Cardiovasc Surg. 1990;99: 798-808.

2. Kitamura S, Mendez A, Kidy JM. Ventricular septal defect following myocardial infarction: experience with surgical repair through a left ventriculotomy and review of literature. J Thorac Cardiovasc Surg. 1971;61:186-99

3. David TE, Dale L, Sun Z. Postinfarction ventricular septal rupture: repair by endocardial patch with infarction exclusion. $J$ Thorac Cardiovasc Surg. 1995;110:1315-22.

4. Jatene AD. Left Ventricular aneurysmectomy: resection or reconstruction. J Thorac Cardiovasc Surg. 1985;89:321-31.

5. Batista R. Partial left ventriculectomy-the Batista procedure. Eur J Cardiothorac Surg. 1999;15(suppl 1):S12-9.

\title{
A case of chest wall angiosarcoma associated with breast implants
}

\author{
Neil D. Saunders, BS, J. Stephen Marshall, MD, and Richard C. Anderson, MD, Peoria, III
}

$\mathrm{P}$ rimary angiosarcomas of the breast or chest wall are exceedingly rare. Generally, primary sarcomas of the breast are less than $0.1 \%$ of breast malignancies, with angiosarcomas making up between $25 \%$ and $40 \%$ of these cases. ${ }^{1}$ Angiosarcomas tend to be aggressive, with a high rate of local recurrence and low 5- and 10-year survival rates. Standard treatment for breast or chest wall angiosarcoma is surgical excision, and there is no clear evidence regarding the role of adjuvant chemotherapy or radiation. ${ }^{2}$ Both irradiation and postmastectomy angioedema are known to substantially increase the risk for angiosarcoma in the breast or chest wall. However, the case we present is primary angiosarcoma in a radiation-naive, augmented breast.

\section{Clinical Summary}

A 58-year-old woman presented with a 4-month history of nonproductive cough and tenderness on the left side of her chest. The patient received bilateral submuscular silicone breast implants in 1975 and had noted that recently the superior aspect of her left breast had become larger than the right. On physical examination, left breast asymmetry was apparent and caused by a firm nonmobile mass. A computed tomographic (CT) scan showed a lobulated

From the Department of Surgery, University of Illinois College of Medicine, Peoria, Peoria, Ill.

Received for publication March 8, 2007; accepted for publication April 23, 2007.

Address for reprints: Neil D. Saunders, 3602 N Kingston Dr, Apt 37, Peoria, IL 61604 (E-mail: neilsaunders@gmail.com).

J Thorac Cardiovasc Surg 2007;134:1076-7

$0022-5223 / \$ 32.00$

Copyright $\odot 2007$ by The American Association for Thoracic Surgery doi:10.1016/j.jtcvs.2007.04.051 mass posterior to her left breast implant measuring $5 \times 4 \mathrm{~cm}$, eroding the third and fourth ribs and extending into the chest (Figure 1). Moderately prominent axillary lymph nodes were also noted. Positron emission tomographic scanning revealed no evidence of spread beyond the anterior left chest wall. Fine-needle aspiration of the mass was pankeratin positive and negative for S100, melanin A, and HMB-45 and was concluded to be nonsmall cell carcinoma.

A wide en bloc excision of the left breast with chest wall and ribs was performed. The tumor was adherent to the pectoralis major and the chest wall (Figure 2). The pectoralis major was removed from the shoulder and clavicle, and the ribs were divided at the sternum. Axillary lymph nodes were removed as well, and the chest wall was reconstructed with a polytetrafluoroethylene* patch. Surgical pathology revealed an epithelioid angiosarcoma staining positive for vimentin, Factor VIII, pankeratin antibody, and CD34. Margins were negative, and no penetration of the pleural space was seen. All 15 of the removed axillary lymph nodes were also negative for primary or metastatic malignancy. The patient did well after the operation and was discharged with a good prognosis.

Five months later, the patient presented with severe anemia and hemoptysis caused by bilateral recurrence of the angiosarcoma. CT of the thorax revealed a $3 \times 3.6-\mathrm{cm}$ mass in the left anterior chest at the level of the aortic arch and bilateral metastatic lung nodules. A left thoracotomy with multiple wedge resection was performed, and the lateral chest wall was further resected. Postoperatively, the patient experienced respiratory failure caused by pneumonia and required mechanical ventilation for 4 weeks. Resection of the right side was unable to be performed. Two months after the second resection, she presented with chest fullness in the left breast area, and a CT scan showed a $10 \times 6-\mathrm{cm}$ left chest wall mass with

*Gore-Tex patch, registered trademark of W. L. Gore \& Associates, Inc, Newark, Del. 


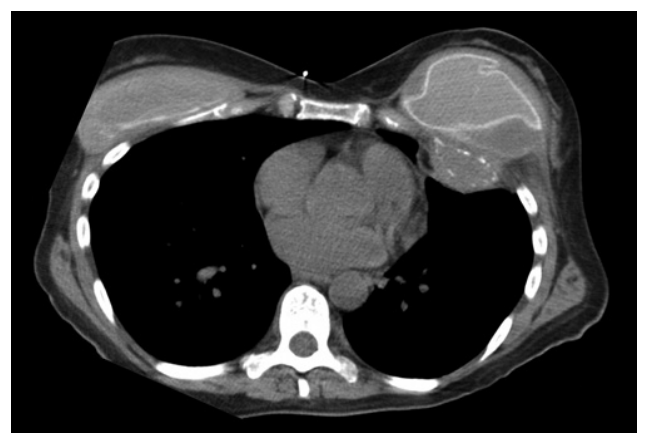

Figure 1. Chest CT scan showing a mass beneath the left breast implant.

extensive left lung nodules. Chemotherapy with paclitaxel (Taxol) was initiated; however, the patient died 16 months later.

\section{Discussion}

For the past 60 years, it has been known that implanted materials can induce inflammation, blood vessel proliferation, and the eventual development of angiosarcoma. In addition to those in animal models, there have been around a dozen cases of angiosarcoma associated with implanted foreign materials, including Dacron aortic grafts, dialysis shunts, and orthopedic implants. ${ }^{3}$ Commonalities among them are long latency periods and high grades at presentation, which is consistent with our case.

Multiple cohort and case-control studies of women with silicone breast implants have shown that implants do not increase one's overall cancer risk, and in many studies the breast cancer rate among women with breast implants is actually lower because of lifestyle variables in women who seek cosmetic surgery. ${ }^{4}$ The types of cancers, sarcoma versus carcinoma, are not broken down in these cohorts, and therefore it is impossible to conclude whether there is an increase in a particular type of cancer. Analysis of the Surveillance Epidemiology and End Results (SEER) data between 1973 and 1990 showed no increase in angiosarcoma in women; however, these data included all women and not just women who received silicone breast implants. ${ }^{5}$

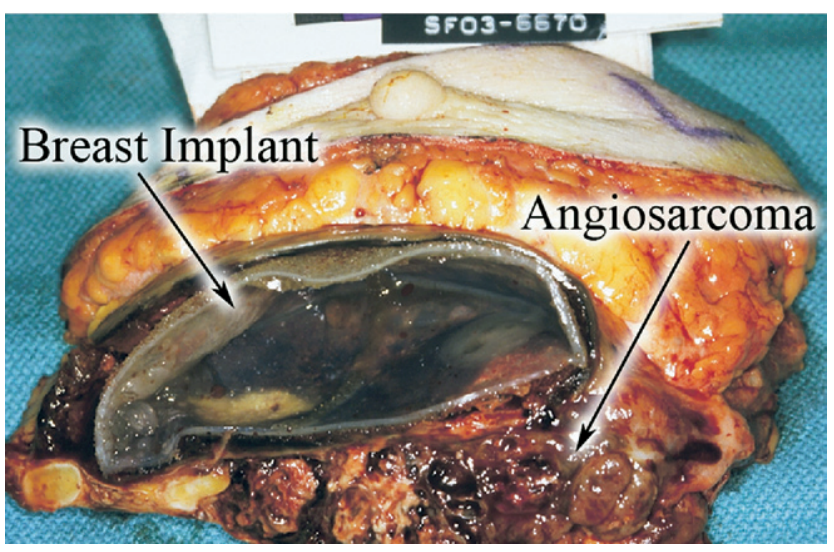

Figure 2. Gross specimen showing implant and angiosarcoma.

The rarity of primary angiosarcoma suggests that even if there was a multifold increase in this cancer among women with breast implants, the resulting data would not have enough power to show a statistically significant change. The miniscule incidence of this particular cancer, including a possible increase caused by implanted materials, would not affect overall breast cancer rates but is a complication of which clinicians should be aware.

\section{References}

1. Adem C, Reynolds C, Ingle JN, Nascimento AG. Primary breast sarcoma: clinicopathologic series from the Mayo Clinic and review of the literature. Br J Cancer. 2004;91:237-41.

2. Moore MP, Kinne DW. Breast sarcoma. Surg Clin North Am. 1996;76: 383-92.

3. Okada M, Takeuchi E, Mori Y, Ichihara S, Usui A, Ueda Y. An autopsy case of angiosarcoma arising around a woven Dacron prosthesis after a Cabrol operation. J Thorac Cardiovasc Surg. 2004;127:1843-5.

4. Brisson J, Holowaty EJ, Villeneuve PJ, Xie L, Ugnat AM, Latulippe L, et al. Cancer incidence in a cohort of Ontario and Quebec women having bilateral breast augmentation. Int J Cancer. 2006;118:2854-62.

5. Engel A, Lamm SH, Lai SH. Human breast sarcoma and human breast implantation: a time trend analysis based on SEER data (1973-1990). J Clin Epidemiol. 1995;48:539-44. 